\title{
Improved Wireless Performance from Mode Scattering in Ventilation Ducts
}

\author{
Benjamin E. Henty* and Daniel D. Stancil \\ Electrical and Computer Engineering Department \\ Carnegie Mellon University, Pittsburgh, PA 15230. \\ henty@eirp.org and stancil@cmu.edu
}

\begin{abstract}
Ventilation ducts are a convenient infrastructure for distributing wireless signals indoors. Conventional and historical use of single conductor metal waveguides for transmission of communications signals have focused on careful excitation and control of the mode content of the waveguidesomething that is possibly infeasible or financially undesirable in a ventilation duct setting. With this in mind we investigate the mode scattering effects present in a ventilation duct T-junction and note with some surprise that improvement in the performance of an IEEE $802.11 \mathrm{~g}$ communication can result from this scattering
\end{abstract}

\section{Introduction}

To facilitate ventilation ducts as an in-building wireless infrastructure, prior work has developed an empirical model of the power loss in a testbed duct system [1]. These results allow a basic link budget calculation for a selection of ventilation duct types. While this is an important factor for designing a ventilation duct based wireless system, the multimoded nature of ventilation duct waveguides should also be considered. In fact, the authors of [1] note a possible impact of mode scattering effects to the power levels measured in test HVAC duct systems. Specifically, they found that the power loss due to a signal that passes through multiple T-junctions is about $9 \mathrm{~dB}$ for the first T-junction and about $3 \mathrm{~dB}$ for each additional T-junction. A $3 \mathrm{~dB}$ loss per junction would be expected if power is divided evenly at the T junction. The authors of [1] hypothesize the additional loss present at the first $\mathrm{T}$-junction encountered was due to a mode filtering effect. So far this hypothesis has yet to be explorered. Although the authors of [2] present a technique for measuring the mode content in straight ducts, they did not attempt T-junction mode content analysis. While several well established techniques exist for mode extraction (see for example [3]), we present here a time domain based technique which can be performed without duct modifications and with only a single pair of antennas yet provides insight into the modal behavior of the ventilation duct channel. In addition, we present IEEE 802.11g throughput measurements in an actual building ventilation duct to investigate the impact that mode filtering has on an actual wireless communication.

\section{Experimental Analysis of Mode Scattering}

To identify the mode scattering phenomenon in a ventilation duct T-junction, a laboratory duct setup as shown in Figure 1 was used. The setup had seven duct

Based in part upon work supported by YIT Building Systems, Inc., and the National Science Foundation under Grant No. 0219278. 
segments of $305 \mathrm{~mm}$ diameter, connected together in a straight run 21.3 meters long with open ends. Quarter wavelength monopole probe antennas, tuned to the 2.4 to $2.5 \mathrm{GHz}$ frequency band, were installed in the duct 20.6 meters apart and a network analyzer was used to characterize the channel response in three different cases. The antennas remained fixed over each of the three cases; only the second duct segment was varied. In the first case the duct segment was unmodified. In the $2^{\text {nd }}$ and $3^{\text {rd }}$ cases a circular hole was cut in the duct segment. In the $2^{\text {nd }}$ case a metal neck, as shown in Figure 2, with a circular, 190 $\mathrm{mm}$ diameter hole (matching the hole cut in the duct) was placed over the hole to form a T-junction representative of the sort used in actual installations. In the 3rd case a metal screen was installed between the metal neck and the duct segment. The metal screen was 18 gage aluminum wire in a $6 \mathrm{~mm}$ square grid. The screen was installed such that it maintained the curvature of the duct and made good electrical contact with the duct and neck.

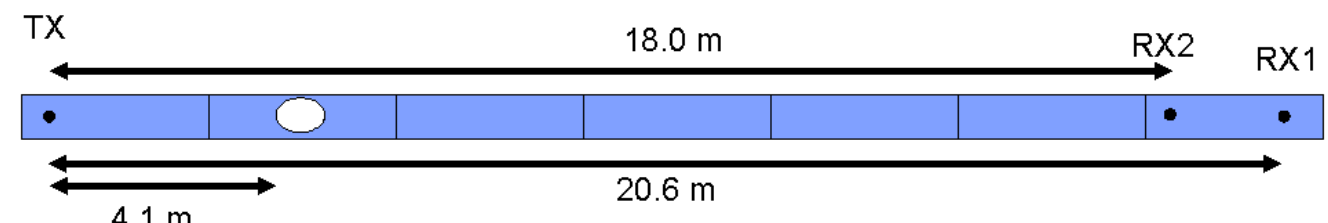

$4.1 \mathrm{~m}$

Figure 1: Schematic of duct section used in experiment.

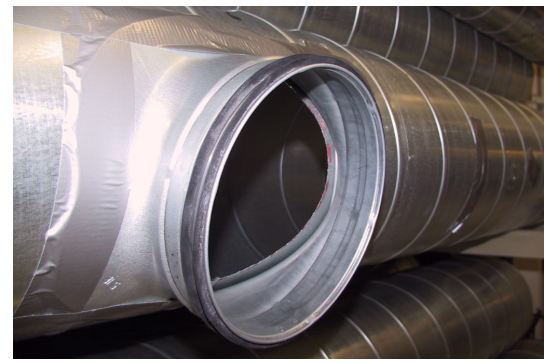

Figure 2: A ventilation duct with a neck of the type used as a T-junction.

The measured frequency responses for the 3 cases are shown in Figure 3a. As can be seen from the frequency response, the screen placed over the hole has nearly an identical response to the unmodified duct case. Further, the loss in power is an average of $7.5 \mathrm{~dB}$ from the unmodified to open hole cases, in reasonable agreement with the $8.9 \mathrm{~dB}$ expected loss, reported in [1]. Using the amplitude and phase of the measured frequency response, the impulse responses of the 3 cases were also computed and are shown in Figure $3 b$.

To analyze the mode content of the responses, the propagation delay of each of the 17 propagating modes was calculated using the theoretical group velocity for propagating modes in a cylindrical waveguide (see for example [4]) and then plotted in Figure 4 with an enlarged portion of the impulse response. The lower order modes appear to bunch up in the early part of the impulse response, while the higher order modes arrive at more spread out intervals. Our results are qualitatively consistent with mode excitation calculations reported in [4] where the most strongly excited modes were found to be $T E_{61}, T E_{51}$, and $T E_{41}$. We now wish to contrast the impulse response of the unmodified and screened hole 

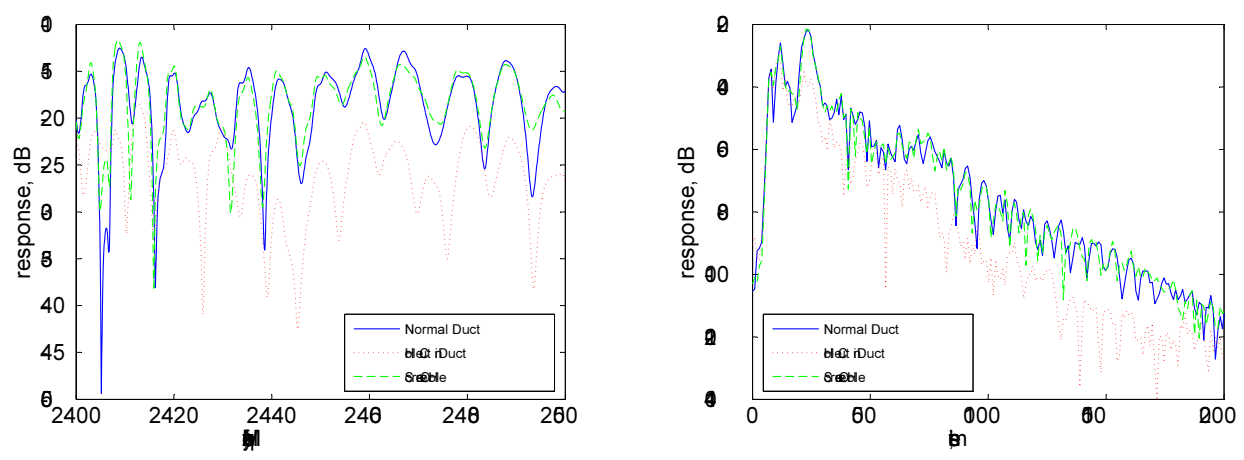

Figure 3: Measured frequency response and corresponding, computed impulse responses for channel measurements of laboratory ventilation duct setup.

cases with the open hole case and observe the mode filtering introduced by the hole cut in the duct. Interestingly, we note that there is very little change in the early arriving power, but a significant decrease in the power of the late arriving modes is observed, particularly the $T E_{61}$. This result is consistent with the hypothesis of [1] in which mode filtering results in an increased power loss at the first T-junction encountered due to mode filtering effects.

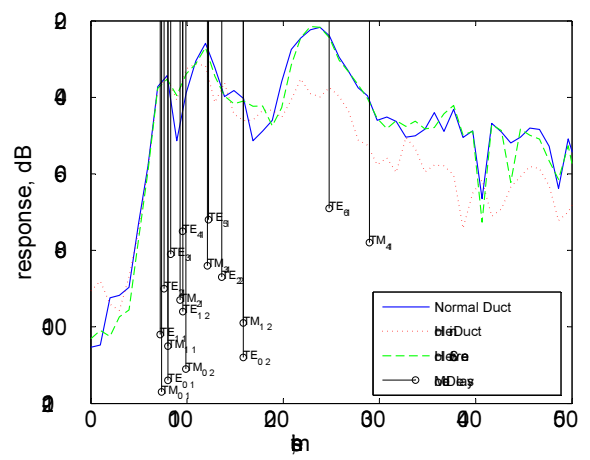

Figure 4: Channel impulse response and computed propagation delays of propagating modes.

\section{Mode Scattering Effect on IEEE 802.11g}

An interesting observation of the filtering effect presented in Figure 3 is that a T-junction appears to reduce the late arriving power and thereby the dispersion and RMS delay spread in the channel. This effect was further explorered in a test installation with an IEEE 802.11g connection in an building ventilation duct. In the test, adapters were used to connect an Access Point and a wireless PCMCIA card to quarter wave monopole antennas inserted approximately 15 meters apart in a length of cylindrical duct $315 \mathrm{~mm}$ in diameter. Five T-junctions of the type measured above were between the two monopoles. Beyond the antenna connected to the PCMCIA card was approximately 5 meters of duct terminated with a flat metal endcap. The 5 meter length of duct had 2 additional T junctions, one to either side of a right angle bend. 0.5 meters beyond the Access Point was a duct 0.3 meter long reduction from $315 \mathrm{~mm}$ to $200 \mathrm{~mm}$ and back to $315 \mathrm{~mm}$ in diameter. After the reduction, there were several tens of meters of duct without any junctions, but with several bends and connected to the main 
Table 1: Performance of IEEE 802.11g through an actual building ventilation duct.

\begin{tabular}{|l|r|r|}
\hline Statistic & Open Holes & Screened Holes \\
\hline \hline Channel RSSI & $-7.2 \mathrm{dBm}$ & $-2.0 \mathrm{dBm}$ \\
Delay Spread & $48 \mathrm{~ns}$ & $105 \mathrm{~ns}$ \\
Throughput & $22.5 \mathrm{Mbps}$ & $8.6 \mathrm{Mbps}$ \\
\hline
\end{tabular}

feed of the building ventilation system. All of the T-junctions had the short neck pieces shown in Figure 2 and a mainly plastic duct louver mechanism mounted to the end of the neck.

Measurements of the signal strength, delay spread and achieved transmit rate and throughput of the IEEE 802.11g test connection were performed for two cases. The first case was the unmodified duct and the second case involved the insertion of metal screens between the necks and the ventilation ducts of all T-junctions. The throughput was measured using Iperf version 1.1.1 [5] configured to use TCP test packets with a 1 megabyte TCP window size. The channel RSSI and delay spread values were computed based on measurements with a network analyzer of the frequency range used by the IEEE $802.11 \mathrm{~g}$ channel. The results presented in Table 1 show that the addition of screens increased the delay spread of the channel and degraded the quality of the communications channel for use with IEEE 802.11g, though the signal strength increased.

\section{Conclusion}

In this work we presented the surprising result that allowing the natural mode filtering present in ventilation duct based wireless distribution systems can be more beneficial than attempting to preserve mode content via airflow compatible metal screens. We have presented measurements of IEEE 802.11g performance in a building's ventilation ducts confirming this result. To better understand the phenomenon, we have used analysis of channel impulse response measurements to identify the mode filtering effect at duct T-junctions, which demonstrates that mode filtering can act to reduce channel delay spread and thereby improve the performance of IEEE $802.11 \mathrm{~g}$ in a ventilation duct system.

We would like to express appreciation to O. Drage and A. Bredesen of YIT Building Systems for making the in-building measurements possible.

\section{References}

[1] O. Z. Tonguz, D. D. Stancil, A. E. Xhafa, A. G. Cepni, P. V. Nikitin, “A Simple Path Loss Prediction Model for HVAC Systems," Proceedings of Globecom, vol. 2, pp. 1850-4, 2002.

[2] P. V. Nikitin, D. D. Stancil, A. G. Cepni, A. E. Xhafa, O. K. Tonguz and D. Brodtkorb, "Novel mode content analysis technique for multimode waveguides," IEEE MTT-S International Microwave Symposium Digest, vol. 3, pp. 1827-30, 2003.

[3] J. M. Baird, D. H. Roper, R. W. Grow, "Surface array waveguide mode analyzer," IEEE MTT-S International Microwave Symposium Digest, vol. 1, pp. 137-140, 1992.

[4] C. A. Balanis, Advanced Engineering Electromagnetics, New York: John Wiley \& Sons, 1989.

[5] Iperf software Internet page available at http://dast.nlanr.net/Projects/Iperf/ 